Rakenteiden Mekaniikka

Vol. 50, Nro 1, 2017, s. $1-16$

http://rakenteidenmekaniikka.journal.fi/index

https://doi.org/10.23998/rm.63299

(C)Kirjoittajat 2017.

Vapaasti saatavilla CC BY-SA 4.0 lisensioitu.

\title{
Venymämitat kontinuumimekaniikassa Hillin-Sethin mukaan
}

\author{
Martti Mikkola
}

Tiivistelmä. Suhteellinen muodonmuutos kontinuumimekaniikassa voidaan määrittää usealla tavalla. R.Hill ja B.R.Seth ovat esittäneet yleisen kaavan, jonka mukaan useat suhteellisen muodonmuutoksen mitat voidaan määrittää. Artikkelissa kyseistä kaavaa sovelletaan tavallisimpiin venymämäärittelyihin mukaan lukien H.Henckyn esittämä logaritminen venymä. Myös venymänopeuksien lausekkeet esitetään. Kuhunkin venymämittaan liittyvä jännitys johdetaan. Sovelluksina tarkastellaan vedettyä sauvaa ja yksinkertaista leikkausta.

Avainsanat: muodonmuutos, yleinen venymämitta, venymänopeus, logaritminen venymä, jännitysmitta.

Vastaanotettu 8.8.2016. Hyväksytty 3.4.201\%. Julkaistu verkossa 11.5.2017.

\section{Johdanto}

Tässä esityksessä vektoreita merkitään vinoilla lihavoiduilla kirjaimilla $\boldsymbol{x}=x^{k} \boldsymbol{g}_{k}=$ $x_{k} \boldsymbol{g}^{k}$ ja niiden dyadituloa $\boldsymbol{x} \otimes \boldsymbol{y}$. Toisen kertaluvun tensoreita merkitään pystyillä lihavoiduilla kirjaimilla $\mathbf{T}=T^{k l} \boldsymbol{g}_{k} \otimes \boldsymbol{g}_{l}=T_{k l} \boldsymbol{g}^{k} \otimes \boldsymbol{g}^{l}$. Kovariantti kantajärjestelmä on $\left\{\boldsymbol{g}_{k}\right\}$ ja kontravariantti vastaavasti $\left\{\boldsymbol{g}^{k}\right\}$. Käytetään myös suorakulmaista yksikkökantajärjestelmää $\left\{\boldsymbol{e}_{K}\right\}$ tai $\left\{\boldsymbol{e}_{k}\right\}$, jonka kovariantit ja kontravariantit kantavektorit tietenkin yhtyvät. Esitystapa noudattaa melko tarkasti G.A. Holzapfelin kirjaa [6].

Tarkastellaan kontinuumikappaletta, joka sijaitsee kolmiulotteisessa euklidisessa avaruudessa. Ainepisteen paikkavektori kiinteässä suorakulmaisessa koordinaatistossa on alkutilassa $\boldsymbol{X}=X^{K} \boldsymbol{e}_{K}$ ja deformoituneessa lopputilassa $\boldsymbol{x}=x^{k}(\boldsymbol{X}, t) \boldsymbol{e}_{k}$. Koordinaattiakselien suuntaiset yksikkökantavektorit ovat $\left\{\boldsymbol{e}_{K}=\boldsymbol{e}_{k}\right\}$. Alku- ja lopputila referoidaan siis samaan karteesiseen koordinaatistoon, mutta alkutilaan viittaavana indeksinä käytetään isoa kirjainta ja lopputilaan viittaavana pientä kirjainta. Deformaatiossa alkutilan koordinaatit $X^{K}$, joita nimitetään materiaalikoordinaateiksi, muodostavat käyräviivaisen koordinaatiston, ks. kuva 1 . Koordinaatit $x^{k}$ ovat nimeltään spatiaalikoordinaatteja. Pisteen $P_{t}$, jonka määrittelevät koordinaatit $\left(X^{1}, X^{2}, X^{3}\right)$, kautta kulkevan koordinaattiviivan yhtälö on muotoa (esim. koordinaattiviiva $\left.X^{1}\right) \boldsymbol{x}=x^{k}\left(s X^{1}, X^{2}, X^{3}, t\right) \boldsymbol{e}_{k}$, jossa $s$ saa reaalisia arvoja. Käyräviivaisen koordinaatiston koordinaattiviivojen tangenttivektorit

$$
\boldsymbol{g}_{K}=\frac{\partial x^{m}}{\partial X^{K}} \boldsymbol{e}_{m}
$$


muodostavat kovariantin kantajärjestelmän $\left\{\boldsymbol{g}_{K}\right\}$. Niissä käytetään indeksinä isoa kirjainta osoittamaan, että ne ovat materiaalisiin koordinaatteihin kuuluvia vektoreita. $\mathrm{Ne}$ eivät tietenkään yleensä ole yksikkövektoreita eivätkä välttämättä myöskään ortogonaalisia. Niitä vastaava resiprookkikanta on

$$
\boldsymbol{g}^{K}=\frac{\partial X^{K}}{\partial x^{m}} \boldsymbol{e}^{m}
$$

Näiden kantavektorien välinen yhteys on

$$
\boldsymbol{g}_{K} \cdot \boldsymbol{g}^{M}=\delta_{M}^{K}
$$

Deformaatiossa alkutilan viiva-alkio $\mathrm{d} \boldsymbol{X}$ kuvautuu viiva-alkioksi $\mathrm{d} \boldsymbol{x}=\mathbf{F} \mathrm{d} \boldsymbol{X}$ deformaatiogradientin $\mathbf{F}$ avulla, ks. kuva 1,

$$
\mathbf{F}=\frac{\partial x^{k}}{\partial X^{M}} \boldsymbol{e}_{k} \otimes \boldsymbol{e}^{M}=\boldsymbol{g}_{M} \otimes \boldsymbol{e}^{M} .
$$

Deformoituneen viiva-alkion pituuden neliö on

$$
\mathrm{d} s^{2}=\mathrm{d} \boldsymbol{x} \cdot \mathrm{d} \boldsymbol{x}=\mathrm{d} \boldsymbol{X} \cdot \mathbf{F}^{\mathrm{T}} \mathbf{F} \mathrm{d} \boldsymbol{X}=\mathrm{d} \boldsymbol{X} \cdot \mathbf{C d} \boldsymbol{X} .
$$

Muodonmuutostensori $\mathbf{C}$ on Cauchyn-Greenin oikeanpuoleinen muodonmuutostensori

$$
\mathbf{C}=\mathbf{F}^{\mathrm{T}} \mathbf{F}=\frac{\partial x^{k}}{\partial X^{M}} \frac{\partial x^{k}}{\partial X^{N}} \boldsymbol{e}^{M} \otimes \boldsymbol{e}^{N}
$$

Vastaavalla tavalla deformoituneen tilan koordinaattiviivat $x^{k}$ esittävät niitä materiaalipartikkeleita, jotka ovat peräisin alkutilan pisteistä $\boldsymbol{X}=X^{K}\left(x^{1}, x^{2}, x^{3}\right) \boldsymbol{e}_{K}$. (Tämä käänteinen yhtälö voidaan muodostaa, sillä $\operatorname{det}(\mathbf{F})>0)$. Ne muodostavat alkutilassa käyräviivaiset koordinaatit, joiden tangenttivektorit ovat

$$
\boldsymbol{G}_{k}=\frac{\partial X^{M}}{\partial x^{k}} \boldsymbol{e}_{M}
$$

Vastaava resiprookkikanta on

$$
\boldsymbol{G}^{k}=\frac{\partial x^{k}}{\partial X^{K}} \boldsymbol{e}^{K}
$$

Nämä kantavektorit toteuttavat yhtälöt

$$
\boldsymbol{G}_{k} \cdot \boldsymbol{G}^{m}=\delta_{m}^{k}
$$

Deformaatiogradientin käänteistensori on

$$
\mathbf{F}^{-1}=\frac{\partial X^{K}}{\partial x^{m}} \boldsymbol{e}_{K} \otimes \boldsymbol{e}^{m}=\boldsymbol{e}_{K} \otimes \boldsymbol{g}^{K}=\boldsymbol{G}_{m} \otimes \boldsymbol{e}^{m} .
$$

Deformaatiogradientin käänteistensori kuvaa deformoituneen tilan viiva-alkion $\mathrm{d} \boldsymbol{x}$ alkutilan viiva-alkioksi $\mathrm{d} \boldsymbol{X}=\mathbf{F}^{-1} \mathrm{~d} \boldsymbol{x}$. Alkutilan viiva-alkion pituuden neliö on

$$
\mathrm{d} S^{2}=\mathrm{d} \boldsymbol{X} \cdot \mathrm{d} \boldsymbol{X}=\mathrm{d} \boldsymbol{x} \cdot \mathbf{F}^{-\mathrm{T}} \mathbf{F}^{-1} \mathrm{~d} \boldsymbol{x}=\mathrm{d} \boldsymbol{x} \cdot \mathbf{b}^{-1} \mathrm{~d} \boldsymbol{x} .
$$

Muodonmuutostensori b on Cauchyn-Greenin vasemmanpuoleinen muodonmuutostensori

$$
\mathbf{b}=\mathbf{F F}^{\mathrm{T}}=\frac{\partial x^{m}}{\partial X^{K}} \frac{\partial x^{n}}{\partial X^{K}} \boldsymbol{e}_{m} \otimes \boldsymbol{e}_{n} .
$$

Huomataan vielä seuraavien yhtälöiden pätevän

$$
\mathbf{F} \boldsymbol{e}_{K}=\boldsymbol{g}_{K}, \quad \mathbf{F}^{-\mathrm{T}} \boldsymbol{e}^{K}=\boldsymbol{g}^{K}, \quad \mathbf{F}^{-1} \boldsymbol{e}_{k}=\boldsymbol{G}_{k}, \quad \mathbf{F}^{\mathrm{T}} \boldsymbol{e}^{k}=\boldsymbol{G}^{k}
$$




\section{Yleistettyjä muodonmuutoksen mittoja}

Muodonmuutostensorit $\mathbf{C}=\mathbf{F}^{\mathrm{T}} \mathbf{F}, \mathbf{U}=\sqrt{\mathbf{C}}$ ja $\mathbf{v}=\mathbf{R} \mathbf{U R}^{\mathrm{T}}$ ovat positiivisesti definiittejä symmetrisiä tensoreita, joten niiden ominaisarvot ovat positiivisia. $\mathbf{R}$ on rotaatiotensori. Merkitään C:n ominaisarvoja (päävenymien neliöitä) $\lambda_{k}^{2}$ :lla ja vastaavia ortonormeerattuja ominaisvektoreita (pääsuuntia) $\hat{\mathbf{N}}_{k}$ :lla. Yllä olevan perusteella voidaan päätellä, että U:n ja v:n ominaisarvot (päävenymät) ovat samat $\lambda_{k}$. Lisäksi nähdään, että U:lla on samat ominaisvektorit kuin C:llä ja että v:n ominaisvektorit ovat $\hat{\boldsymbol{n}}_{k}=\mathbf{R} \hat{\boldsymbol{N}}_{k}$.

Seth [8] ja Hill [3] ovat esittäneet yleiseksi venymämitan muodoksi

$$
\begin{gathered}
\mathbf{E}=f(\mathbf{U})=\sum_{K=1}^{3} f\left(\lambda_{k}\right) \hat{\boldsymbol{N}}_{k} \otimes \hat{\boldsymbol{N}}_{k}, \\
\mathbf{e}=f(\mathbf{v})=\sum_{k=1}^{3} f\left(\lambda_{k}\right) \hat{\boldsymbol{n}}_{k} \otimes \hat{\boldsymbol{n}}_{k} .
\end{gathered}
$$

Venytystensorit $\mathbf{U}$ ja $\mathbf{v}$ ovat Lagrangen ja Eulerin esitystapojen mukaiset venymät. Funktiota $f$ nimitetään skaalausfunktioksi, joka on monotonisesti kasvava ja toteuttaa ehdot $f(1)=0, f^{\prime}(1)=1$. Seth ja Hill ovat esittäneet skaalausfunktioksi

$$
f(\lambda)=\frac{1}{m}\left(\lambda^{m}-1\right), m \neq 0, \quad f(\lambda)=\ln \lambda, m=0 .
$$

Tätä skaalausfunktiota käyttäen kaavat ( 14 ) ja (15) johtavat muotoihin

$$
\begin{aligned}
\mathbf{E}^{(m)} & =\frac{1}{m}\left(\mathbf{U}^{m}-\mathbf{I}\right), \\
\mathbf{e}^{(m)} & =\frac{1}{m}\left(\mathbf{v}^{m}-\mathbf{i}\right) .
\end{aligned}
$$

Alkutilan identiteettitensori on $\mathbf{I}=\hat{\boldsymbol{N}}_{k} \otimes \hat{\boldsymbol{N}}_{k}$ ja deformoituneen tilan $\mathbf{i}=\hat{\boldsymbol{n}}_{k} \otimes \hat{\boldsymbol{n}}_{k}$.

Lagrangen esitystavan mukaiset venymämitat ovat silloin

$$
\begin{aligned}
& \text { Greenin-Lagrangen venymä: } & \mathbf{E}^{(2)} & =\frac{1}{2}\left(\mathbf{U}^{2}-\mathbf{I}\right)=\frac{1}{2}\left(\mathbf{F}^{\mathrm{T}} \mathbf{F}-\mathbf{I}\right), \\
& \text { Almansin-Eulerin venymä: } & \mathbf{E}^{(-2)} & =\frac{1}{2}\left(\mathbf{I}-\mathbf{U}^{-2}\right)=\mathbf{R}^{\mathrm{T}} \frac{1}{2}\left(\mathbf{i}-\mathbf{F}^{-\mathrm{T}} \mathbf{F}^{-1}\right) \mathbf{R}, \\
& \text { Biot'n venymä: } & \mathbf{U}_{B} & =\mathbf{E}^{(1)}=\mathbf{U}-\mathbf{I}=\sum_{K=1}^{3}\left(\lambda_{k}-1\right) \hat{\mathbf{N}}_{k} \otimes \hat{\mathbf{N}}_{k}, \\
& \text { Henckyn venymä: } & \mathbf{E}^{(0)} & =\ln \mathbf{U}=\sum_{K=1}^{3}\left(\ln \lambda_{k}\right) \hat{\mathbf{N}}_{k} \otimes \hat{\mathbf{N}}_{k} .
\end{aligned}
$$

Uusi venymämitta, Henckyn venymä $\mathbf{E}^{(0)}[2]$, on siis logaritminen vastaten yksidimensioista muotoa $e_{\text {log }}=\ln \left(l / l_{0}\right)$. 


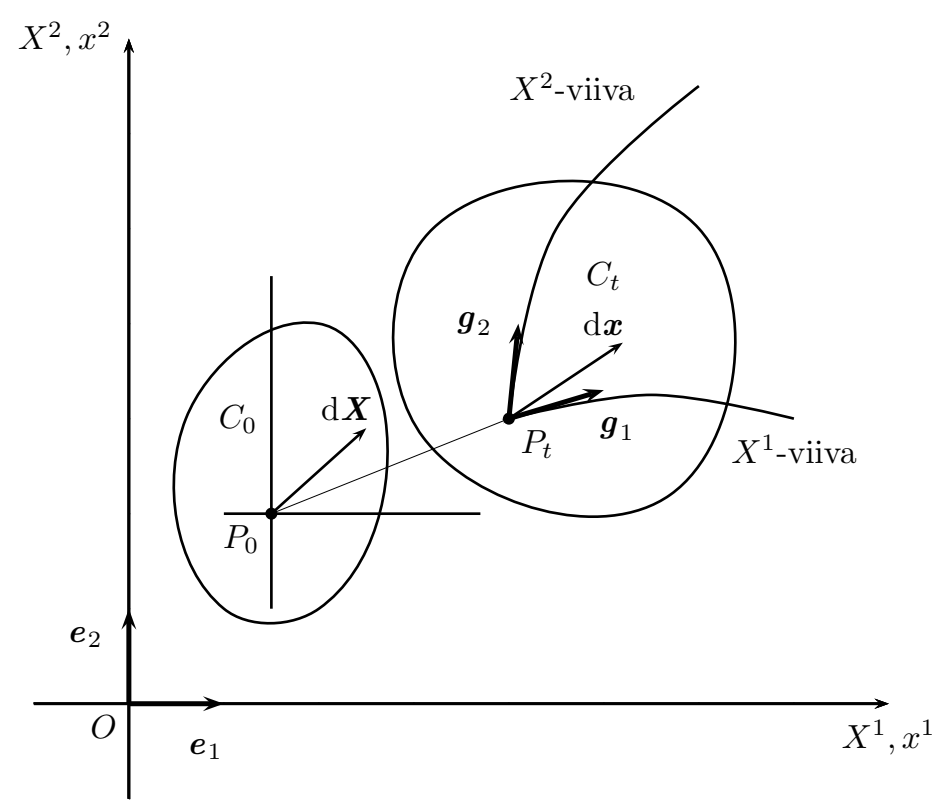

Kuva 1. Koordinaatisto ja alkutila $C_{0}$ sekä lopputila $C_{t}$.

Eulerin esitystavassa edellä esitetyt venymät ovat

Greenin-Lagrangen venymä:

$$
\mathbf{e}^{(2)}=\mathbf{R E}^{(2)} \mathbf{R}^{\mathrm{T}}=\frac{1}{2}\left(\mathbf{v}^{2}-\mathbf{i}\right)
$$

Almansin-Eulerin venymä:

$\mathbf{e}^{(-2)}=\mathbf{R} \mathbf{E}^{(-2)} \mathbf{R}^{\mathrm{T}}=\frac{1}{2}\left(\mathbf{i}-\mathbf{v}^{-2}\right)$,

Biot'n venymä:

$\mathbf{v}_{B}=\mathbf{e}^{(1)}=\mathbf{v}-\mathbf{i}=\mathbf{R} \mathbf{E}^{(1)} \mathbf{R}^{\mathrm{T}}$,

Henckyn venymä:

$$
\mathbf{e}^{(0)}=\ln \mathbf{v}=\sum_{k=1}^{3}\left(\ln \lambda_{k}\right) \hat{\boldsymbol{n}}_{k} \otimes \hat{\boldsymbol{n}}_{k}=\mathbf{R} \mathbf{E}^{(0)} \mathbf{R}^{\mathrm{T}}
$$

Logaritmista venymää kutsutaan myös luonnolliseksi tai todelliseksi venymäksi. Tämä johtunee siitä, että vetokokeessa todellisen jännityksen $\sigma$ ja venymänopeuden $\dot{e}_{l o g}$ tulo $\sigma \dot{e}_{l o g}$ on jännityksen teho. Logaritminen muodonmuutostensori on käyttökelpoinen konstitutiivisten mallien yhteydessä, sillä se voidaan jakaa helposti additiivisesti tilavuudenmuutosja deviatoriseen osaan [6].

\section{Polaarihajoitelma ja nopeusgradientti}

Deformaatiogradientti ja sen käänteisoperaattori voidaan esittää polaarihajoitelman avulla

$$
\mathbf{F}=\mathbf{R U}=\mathbf{v R}=\sum_{k=1}^{3} \lambda_{k} \hat{\boldsymbol{n}}_{k} \otimes \hat{\boldsymbol{N}}_{k}, \mathbf{F}^{-1}=\mathbf{U}^{-1} \mathbf{R}^{\mathrm{T}}=\sum_{k=1}^{3} \lambda_{k}^{-1} \hat{\boldsymbol{N}}_{K} \otimes \hat{\boldsymbol{n}}_{k} .
$$

Rotaatiotensori $\mathbf{R}$ ja sen nopeus ovat muotoa

$$
\mathbf{R}=\sum_{k=1}^{3} \hat{\boldsymbol{n}}_{k} \otimes \hat{\boldsymbol{N}}_{k}, \boldsymbol{\Omega}^{(\mathrm{R})}=\dot{\mathbf{R}} \mathbf{R}^{\mathrm{T}}
$$


Antisymmetrinen tensori $\boldsymbol{\Omega}^{(\mathrm{L})}$, joka määrittää ominaisvektorien $\hat{\boldsymbol{N}}_{k}$ muutosnopeuden, saadaan seuraavasti. Ominaisvektori on muotoa

$$
\hat{\mathbf{N}}_{k}=\mathbf{R}^{(\mathrm{L})} \boldsymbol{e}_{k}
$$

jossa $\left\{\boldsymbol{e}_{k}\right\}$ on kiinteä kantajärjestelmä ja $\mathbf{R}^{(\mathrm{L})}$ rotaatiomatriisi niin että $\left(\mathbf{R}^{(\mathrm{L})}\right)^{-1}=$ $\left(\mathbf{R}^{(\mathrm{L})}\right)^{\mathrm{T}}$. Ominaisvektorin aikaderivaatta on silloin

$$
\begin{aligned}
\dot{\hat{\boldsymbol{N}}}_{k}=\dot{\mathbf{R}}^{(\mathrm{L})} \boldsymbol{e}_{k}=\dot{\mathbf{R}}^{(\mathrm{L})}\left(\mathbf{R}^{(\mathrm{L})}\right)^{-1} \hat{\boldsymbol{N}}_{k}=\boldsymbol{\Omega}^{(\mathrm{L})} \hat{\boldsymbol{N}}_{k} & \\
& \Rightarrow \boldsymbol{\Omega}^{(\mathrm{L})}=\dot{\mathbf{R}}^{(\mathrm{L})}\left(\mathbf{R}^{(\mathrm{L})}\right)^{-1}=\dot{\mathbf{R}}^{(\mathrm{L})}\left(\mathbf{R}^{(\mathrm{L})}\right)^{\mathrm{T}}
\end{aligned}
$$

Vastaavasti ominaisvektorin $\hat{\boldsymbol{n}}_{k}=\mathbf{R} \hat{\boldsymbol{N}}_{\boldsymbol{k}}$ aikaderivaatta on

$$
\dot{\hat{\boldsymbol{n}}}_{k}=\mathbf{R} \dot{\hat{\boldsymbol{N}}}_{k}+\dot{\mathbf{R}} \hat{\boldsymbol{N}}_{k}=\mathbf{R} \boldsymbol{\Omega}^{(\mathrm{L})} \mathbf{R}^{\mathrm{T}} \hat{\boldsymbol{n}}_{k}+\boldsymbol{\Omega}^{(\mathrm{R})} \hat{\boldsymbol{n}}_{k}=\boldsymbol{\Omega}^{(\mathrm{E})} \hat{\boldsymbol{n}}_{k} .
$$

Rotaationopeuksien välinen riippuvuus on kiertyneessä ominaisvektorikannassa $\left\{\hat{\boldsymbol{n}}_{k}\right\}$

$$
\boldsymbol{\Omega}^{(\mathrm{E})}=\boldsymbol{\Omega}^{(\mathrm{R})}+\mathbf{R} \boldsymbol{\Omega}^{(\mathrm{L})} \mathbf{R}^{(\mathrm{T})} .
$$

Deformaatiogradientin aikaderivaatta on

$$
\dot{\mathbf{F}}=\sum_{k=1}^{3} \dot{\lambda}_{k} \hat{\boldsymbol{n}}_{k} \otimes \hat{\boldsymbol{N}}_{k}+\boldsymbol{\Omega}^{(\mathrm{E})} \mathbf{F}-\mathbf{F} \boldsymbol{\Omega}^{(\mathrm{L})}
$$

Nopeusgradientti saa silloin muodon

$$
\begin{aligned}
\mathbf{L}=\dot{\mathbf{F}} \mathbf{F}^{-1}=\sum_{k=1}^{3} \dot{\lambda}_{k} \lambda_{k}^{-1} \hat{\boldsymbol{n}}_{k} \otimes \hat{\boldsymbol{n}}_{k} & +\boldsymbol{\Omega}^{(\mathrm{E})}-\mathbf{F} \boldsymbol{\Omega}^{(\mathrm{L})} \mathbf{F}^{-1} \\
& =\sum_{k=1}^{3} \dot{\lambda}_{k} \lambda_{k}^{-1} \hat{\boldsymbol{n}}_{k} \otimes \hat{\boldsymbol{n}}_{k}+\boldsymbol{\Omega}^{(\mathrm{E})}-\mathbf{v}\left(\boldsymbol{\Omega}^{(\mathrm{E})}-\boldsymbol{\Omega}^{(\mathrm{R})}\right) \mathbf{v}^{-1} .
\end{aligned}
$$

Venymänopeuden D lausekkeeksi tulee tällöin

$$
\mathbf{D}=\frac{1}{2}\left(\mathbf{L}+\mathbf{L}^{\mathrm{T}}\right)=\sum_{k=1}^{3} \dot{\lambda}_{k} \lambda_{k}^{-1} \hat{\boldsymbol{n}}_{k} \otimes \hat{\boldsymbol{n}}_{k}-\frac{1}{2}\left(\mathbf{F} \boldsymbol{\Omega}^{(\mathrm{L})} \mathbf{F}^{-1}-\mathbf{F}^{-\mathrm{T}} \boldsymbol{\Omega}^{(\mathrm{L})} \mathbf{F}^{\mathrm{T}}\right) .
$$

Kiertymänopeus $\mathbf{W}$ on vastaavasti

$$
\mathbf{W}=\frac{1}{2}\left(\mathbf{L}-\mathbf{L}^{\mathrm{T}}\right)=\boldsymbol{\Omega}^{(\mathrm{E})}-\frac{1}{2}\left(\mathbf{F} \boldsymbol{\Omega}^{(\mathrm{L})} \mathbf{F}^{-1}+\mathbf{F}^{-\mathrm{T}} \boldsymbol{\Omega}^{(\mathrm{L})} \mathbf{F}^{\mathrm{T}}\right) .
$$

Näiden komponentit $\left\{\hat{\boldsymbol{n}}_{k}\right\}$-kannassa ovat

$$
\begin{gathered}
D_{k k}=\dot{\lambda}_{k} \lambda_{k}^{-1}, D_{i k}=\left(\Omega_{i k}^{(\mathrm{E})}-\Omega_{i k}^{(\mathrm{R})}\right) \frac{\lambda_{k}^{2}-\lambda_{i}^{2}}{2 \lambda_{k} \lambda_{i}}=\Omega_{i k}^{(\mathrm{L})} \frac{\lambda_{k}^{2}-\lambda_{i}^{2}}{2 \lambda_{k} \lambda_{i}},(k, l=1,2,3, \text { ei summ. }), \quad(29) \\
W_{k k}=0, W_{i k}=\Omega_{i k}^{(\mathrm{E})}-\left(\Omega_{i k}^{(\mathrm{E})}-\Omega_{i k}^{(\mathrm{R})}\right) \frac{\lambda_{k}^{2}+\lambda_{i}^{2}}{2 \lambda_{k} \lambda_{i}}=\Omega_{i k}^{(\mathrm{E})}-\Omega_{i k}^{(\mathrm{L})} \frac{\lambda_{k}^{2}+\lambda_{i}^{2}}{2 \lambda_{k} \lambda_{i}},(k, l=1,2,3, \text { ei summ. }) .
\end{gathered}
$$




\section{Venymänopeuden lausekkeet}

Venymän muodoista (14) ja (15) venymänopeuksien lausekkeiksi saadaan

$$
\dot{\mathbf{E}}=\sum_{k=1}^{3} f^{\prime}\left(\lambda_{k}\right) \dot{\lambda}_{k} \hat{\boldsymbol{N}}_{k} \otimes \hat{\boldsymbol{N}}_{k}+\boldsymbol{\Omega}^{(\mathrm{L})} \mathbf{E}-\mathbf{E} \boldsymbol{\Omega}^{(\mathrm{L})}
$$

ja

$$
\dot{\mathbf{e}}=\sum_{k=1}^{3} f^{\prime}\left(\lambda_{k}\right) \dot{\lambda}_{k} \hat{\boldsymbol{n}}_{k} \otimes \hat{\boldsymbol{n}}_{k}+\boldsymbol{\Omega}^{(\mathrm{E})} \mathbf{e}-\mathbf{e} \boldsymbol{\Omega}^{(\mathrm{E})} .
$$

Vertaamalla edellä olevien lausekkeiden summatermejä nähdään helposti, että

$$
\dot{\mathrm{e}}-\boldsymbol{\Omega}^{(\mathrm{E})} \mathbf{e}+\mathbf{e} \boldsymbol{\Omega}^{(\mathrm{E})}=\mathbf{R}\left[\dot{\mathbf{E}}-\boldsymbol{\Omega}^{(\mathrm{L})} \mathbf{E}+\mathbf{E} \boldsymbol{\Omega}^{(\mathrm{L})}\right] \mathbf{R}^{\mathrm{T}} .
$$

Lagrangen esitystavan mukaiset venymänopeudet ovat

Green-Lagrange: $\quad \dot{\mathbf{E}}^{(\mathbf{2})}=\sum_{k=1}^{3} \lambda_{k} \dot{\lambda}_{k} \hat{\boldsymbol{N}}_{k} \otimes \hat{\boldsymbol{N}}_{k}+\mathbf{\Omega}^{(\mathrm{L})} \mathbf{E}^{(\mathbf{2})}-\mathbf{E}^{(\mathbf{2})} \mathbf{\Omega}^{(\mathrm{L})}$,

Almansi-Euler: $\quad \dot{\mathbf{E}}^{(-\mathbf{2})}=\sum_{k=1}^{3} \lambda_{k}^{-3} \dot{\lambda}_{k} \hat{\boldsymbol{N}}_{k} \otimes \hat{\boldsymbol{N}}_{k}+\boldsymbol{\Omega}^{(\mathrm{L})} \mathbf{E}^{(-\mathbf{2})}-\mathbf{E}^{(-\mathbf{2})} \boldsymbol{\Omega}^{(\mathrm{L})}$,

Biot:

$$
\dot{\mathbf{U}}=\dot{\mathbf{E}}^{(1)}=\sum_{k=1}^{3} \dot{\lambda}_{k} \hat{\boldsymbol{N}}_{k} \otimes \hat{\boldsymbol{N}}_{k}+\boldsymbol{\Omega}^{(\mathrm{L})} \mathbf{E}^{(\mathbf{1})}-\mathbf{E}^{(\mathbf{1})} \boldsymbol{\Omega}^{(\mathrm{L})},
$$

Hencky: $\quad \dot{\mathbf{E}}^{(0)}=(\ln \mathbf{U})^{\bullet}=\sum_{k=1}^{3} \lambda_{k}^{-1} \dot{\lambda}_{k} \hat{\boldsymbol{N}}_{k} \otimes \hat{\boldsymbol{N}}_{k}+\boldsymbol{\Omega}^{(\mathrm{L})} \mathbf{E}^{(\mathbf{0})}-\mathbf{E}^{(\mathbf{0})} \boldsymbol{\Omega}^{(\mathrm{L})}$.

Eulerin esitystavan mukaiset venymänopeudet ovat vastaavasti

$$
\begin{array}{llrl} 
& \text { Green-Lagrange: } & \dot{\mathbf{e}}^{(\mathbf{2})} & =\sum_{k=1}^{3} \lambda_{k} \dot{\lambda}_{k} \hat{\boldsymbol{n}}_{k} \otimes \hat{\boldsymbol{n}}_{k}+\boldsymbol{\Omega}^{(\mathrm{E})} \mathbf{e}^{(\mathbf{2})}-\mathbf{e}^{(\mathbf{2})} \boldsymbol{\Omega}^{(\mathrm{E})}, \\
& \text { Almansi-Euler: } & \dot{\mathbf{e}}^{(-\mathbf{2})} & =\sum_{k=1}^{3} \lambda_{k}^{-3} \dot{\lambda}_{k} \hat{\boldsymbol{n}}_{k} \otimes \hat{\boldsymbol{n}}_{k}+\boldsymbol{\Omega}^{(\mathrm{E})} \mathbf{e}^{(-2)}-\mathbf{e}^{(-2)} \boldsymbol{\Omega}^{(\mathrm{E})}, \\
& \text { Biot: } & \dot{\mathbf{e}}^{(1)} & =\sum_{k=1}^{3} \dot{\lambda}_{k} \hat{\boldsymbol{n}}_{k} \otimes \hat{\boldsymbol{n}}_{k}+\boldsymbol{\Omega}^{(\mathrm{E})} \mathbf{e}^{(1)}-\mathbf{e}^{(1)} \boldsymbol{\Omega}^{(\mathrm{E})}, \\
& \text { Hencky: } & \dot{\mathbf{e}}^{(0)} & =(\ln \mathbf{v})^{\bullet}=\sum_{k=1}^{3} \lambda_{k}^{-1} \dot{\lambda}_{k} \hat{\boldsymbol{n}}_{k} \otimes \hat{\boldsymbol{n}}_{k}+\boldsymbol{\Omega}^{(\mathrm{E})} \mathbf{e}^{(0)}-\mathbf{e}^{(0)} \boldsymbol{\Omega}^{(\mathrm{E})} .
\end{array}
$$

\section{Jännitysten ja venymien vastaavuus}

Seuraavassa johdetaan jännitysmittojen lausekkeet Kirchhoffin (tai Cauchyn) jännityksen funktioina. Jännitysten ja venymien vastaavuus seuraa jännitysten tehon yhtäsuuruudesta

$$
\mathbf{T}^{(n)}: \mathbf{E}^{(n)}=J \boldsymbol{\sigma}: \mathbf{D}=\boldsymbol{\Sigma}: \mathbf{D}=\Sigma_{k l} D_{k l} .
$$

Edellä $\boldsymbol{\Sigma}=J \boldsymbol{\sigma}$ on Kirchhoffin jännitystensori. Komponentit ovat kannan $\left\{\hat{\boldsymbol{n}}_{k}\right\}$ suhteen. 
Kaavan $(33)_{1}$ mukaan saadaan aluksi

$\boldsymbol{\Omega}^{(\mathrm{L})} \mathbf{E}^{(2)}=\frac{1}{2} \sum_{k, l=1}^{3} \Omega_{k l}^{(\mathrm{L})}\left(\lambda_{l}^{2}-1\right) \hat{\boldsymbol{N}}_{k} \otimes \hat{\boldsymbol{N}}_{l}, \quad$ ja $\quad \mathbf{E}^{(2)} \boldsymbol{\Omega}^{(\mathrm{L})}=\frac{1}{2} \sum_{k, l=1}^{3} \Omega_{k l}^{(\mathrm{L})}\left(\lambda_{k}^{2}-1\right) \hat{\boldsymbol{N}}_{k} \otimes \hat{\boldsymbol{N}}_{l}$,

minkä jälkeen saadaan

$$
\dot{\mathbf{E}}^{(2)}=\sum_{k=1}^{3} f^{\prime}\left(\lambda_{k}\right) \dot{\lambda}_{k} \hat{\boldsymbol{N}}_{k} \otimes \hat{\boldsymbol{N}}_{k}+\frac{1}{2} \sum_{k, l=1}^{3} \Omega_{k l}^{(\mathrm{L})}\left(\lambda_{l}^{2}-\lambda_{k}^{2}\right) \hat{\mathbf{N}}_{k} \otimes \hat{\mathbf{N}}_{l}
$$

Sen jälkeen muodostetaan kaavan (35) mukaan lauseke

$$
\mathbf{T}^{(2)}: \dot{\mathbf{E}}^{(2)}=\sum_{k=1}^{3} T_{k k}^{(2)} \lambda_{k} \dot{\lambda}_{k}+\frac{1}{2} \sum_{k, l=1, k \neq l}^{3} T_{k l}^{(2)} \Omega_{k l}^{(\mathrm{L})}\left(\lambda_{l}^{2}-\lambda_{k}^{2}\right) .
$$

Jännitystensori $\mathbf{T}^{(2)}$ on Piolan-Kirchhoffin toinen jännitys. Vertaamalla tätä kaavoihin (29) ja (35) nähdään, että

$$
T_{k l}^{(2)}=\Sigma_{k l} /\left(\lambda_{k} \lambda_{l}\right), \quad(k, l=1,2,3, \text { ei summausta }) .
$$

Sama tulos seuraa myös yhteydestä $\mathbf{T}^{(2)}=\mathbf{F}^{-1} \mathbf{\Sigma} \mathbf{F}^{-\mathrm{T}}$. Jännityskomponentit $T_{k l}^{(2)}$ ovat kannan $\left\{\hat{\boldsymbol{N}}_{k}\right\}$ suhteen.

Almansin-Eulerin venymää vastaava jännitys seuraa yhtälöstä

$$
\mathbf{T}^{(-2)}: \dot{\mathbf{E}}^{(-2)}=\sum_{k=1}^{3} T_{k k}^{(-2)} \lambda_{k}^{-3} \dot{\lambda}_{k}+\frac{1}{2} \sum_{k, l=1, k \neq l}^{3} T_{k l}^{(-2)} \Omega_{k l}^{(\mathrm{L})}\left(\lambda_{k}^{-2}-\lambda_{l}^{-2}\right)
$$

joka antaa yhteyden

$$
T_{k l}^{(-2)}=\Sigma_{k l} \lambda_{k} \lambda_{l}, \quad(k, l=1,2,3, \text { ei summausta })
$$

Sama tulos saadaan yhteydestä $\mathbf{T}^{(-2)}=\mathbf{F}^{\mathrm{T}} \boldsymbol{\Sigma} \mathbf{F}$. Jännityskomponentit $T_{k l}^{(-2)}$ ovat kannan $\left\{\hat{\mathbf{N}}_{k}\right\}$ suhteen.

Biot'n jännityksen symmetrisen osan ja vastaavan venymän välinen yhteys seuraa yhtälöstä

$$
\mathbf{T}^{(1)}: \dot{\mathbf{E}}^{(\mathbf{1})}=\sum_{k=1}^{3} T_{k k}^{(1)} \dot{\lambda}_{k}+\sum_{k, l=1, k \neq l}^{3} T_{k l}^{(1)} \Omega_{k l}^{(\mathrm{L})}\left(\lambda_{l}-\lambda_{k}\right)
$$

Vertaamalla taas kaavoihin (29) ja (35) nähdään Biot'n ja Kirchhoffin jännitysten välinen yhteys

$$
T_{k k}^{(1)}=\Sigma_{k k} / \lambda_{k}, \quad T_{k l}^{(1)}=\frac{1}{2} \Sigma_{k l}\left(1 / \lambda_{k}+1 / \lambda_{l}\right), \quad(k, l=1,2,3, \text { ei summausta }) .
$$

Sama tulos saadaan myös Biot'n jännityksen symmetrisen osan ja Piolan-Kirchhoffin toisen jännityksen välisestä yhteydestä $\mathbf{T}^{(1)}=\frac{1}{2}\left(\mathbf{U} \mathbf{T}^{(2)}+\mathbf{T}^{(2)} \mathbf{U}\right)$.

Piolan-Kirchhoffin ensimmäisen jännityksen $\mathbf{P}$ ja Cauchyn jännityksen välinen yhteys saadaan helposti käyttämällä yhtälöä $\mathbf{P}=\mathbf{F} \mathbf{T}^{(2)}$

$$
P_{k l}=\lambda_{k} T_{k l}^{(2)}=\Sigma_{k l} / \lambda_{l}=J \sigma_{k l} / \lambda_{l}, \quad(k, l=1,2,3, \text { ei summausta }) .
$$




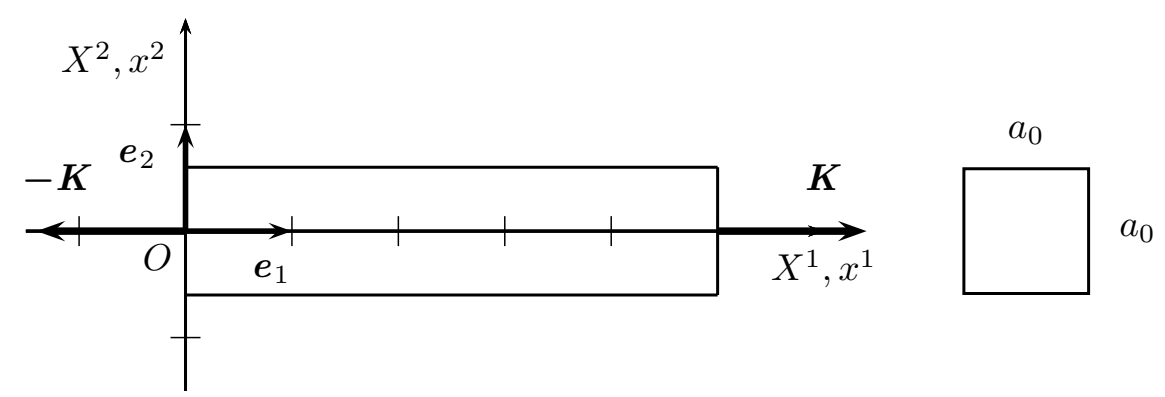

Kuva 2. Vedetty sauva.

Tässä ensimmäinen alaindeksi viittaa kantaan $\left\{\hat{\boldsymbol{n}}_{k}\right\}$ ja jälkimmäinen kantaan $\left\{\hat{\boldsymbol{N}}_{k}\right\}$.

Logaritmista venymää vastaava jännitys saadaan muodostamalla kaavaa (35) käyttäen lauseke

$$
\mathbf{T}^{(0)}: \dot{\mathbf{E}}^{(0)}=\sum_{k=1}^{3} T_{k k}^{(0)} \lambda_{k}^{-1} \dot{\lambda}_{k}+\sum_{k, l=1, k \neq l}^{3} T_{k l}^{(0)} \Omega_{k l}^{(\mathrm{L})}\left(\ln \lambda_{l}-\ln \lambda_{k}\right) .
$$

Vertaamalla kaavoihin (29) ja (35) nähdään, että

$$
\begin{aligned}
T_{k k}^{(0)}=\Sigma_{k k}, T_{k l}^{(0)}=\Sigma_{k l}\left(\lambda_{l}^{2}-\lambda_{k}^{2}\right) /\left[2 \lambda_{k} \lambda_{l} \ln \left(\lambda_{l} / \lambda_{k}\right)\right] & \\
& \left(k \neq l, \lambda_{k} \neq \lambda_{l}\right),(k, l=1,2,3, \text { ei summausta }) .
\end{aligned}
$$

Tässä on käsitelty vain tapausta, jossa ominaisarvot ovat eri suuria. Mm. Macvean [7], Hoger [4], [5] ja Gurtin [1] ovat tarkastelleet myös muita tapauksia.

\section{Esimerkki 1. Vedetty sauva}

Otaksutaan, että karteesisen koordinaatiston $X^{1}$-akseli yhtyy sauvan akseliin. Kantavektoreita merkitään $\left\{\mathbf{e}_{k}\right\}$. Deformaatio on muotoa $x^{1}=\lambda_{1} X^{1}, x^{2}=\lambda X^{2}, x^{3}=\lambda X^{3}$, jossa positiiviset luvut $\lambda_{1}$ ja $\lambda$ kuvaavat venytyksiä koordinaattiakselien suunnissa. Silloin deformaatiogradientti on $\mathbf{F}=\lambda_{1} \boldsymbol{e}_{1} \otimes \boldsymbol{e}^{1}+\lambda \boldsymbol{e}_{2} \otimes \boldsymbol{e}^{2}+\lambda \boldsymbol{e}_{3} \otimes \boldsymbol{e}^{3}$. Polaarihajoitelman rotaatiotensori on yksikkötensori, joten $\mathbf{F}=\mathbf{U}=\mathbf{v}$. Muodonmuutoksen pääsuunnat yhtyvät koordinaattiakselien suuntiin, joten $\hat{\boldsymbol{N}}_{k}=\hat{\boldsymbol{n}}_{k}=\boldsymbol{e}_{k}$, (kuva 2).

Muodonmuutostensorit ovat

$$
\begin{aligned}
\mathbf{E}^{(2)} & =\frac{1}{2}\left(\lambda_{1}^{2}-1\right) \boldsymbol{e}_{1} \otimes \boldsymbol{e}_{1}+\frac{1}{2}\left(\lambda^{2}-1\right)\left(\boldsymbol{e}_{2} \otimes \boldsymbol{e}_{2}+\boldsymbol{e}_{3} \otimes \boldsymbol{e}_{3}\right) \\
\mathbf{E}^{(-2)} & =\mathbf{e}^{(-2)}=\frac{1}{2}\left(1-\lambda_{1}^{-2}\right) \boldsymbol{e}_{1} \otimes \boldsymbol{e}_{1}+\frac{1}{2}\left(1-\lambda^{-2}\right)\left(\boldsymbol{e}_{2} \otimes \boldsymbol{e}_{2}+\boldsymbol{e}_{3} \otimes \boldsymbol{e}_{3}\right) \\
\mathbf{E}^{(1)} & =\left(\lambda_{1}-1\right) \boldsymbol{e}_{1} \otimes \boldsymbol{e}_{1}+(\lambda-1)\left(\boldsymbol{e}_{2} \otimes \boldsymbol{e}_{2}+\boldsymbol{e}_{3} \otimes \boldsymbol{e}_{3}\right) \\
\mathbf{E}^{(0)} & =\ln \lambda_{1} \boldsymbol{e}_{1} \otimes \boldsymbol{e}_{1}+\ln \lambda\left(\boldsymbol{e}_{2} \otimes \boldsymbol{e}_{2}+\boldsymbol{e}_{3} \otimes \boldsymbol{e}_{3}\right)
\end{aligned}
$$




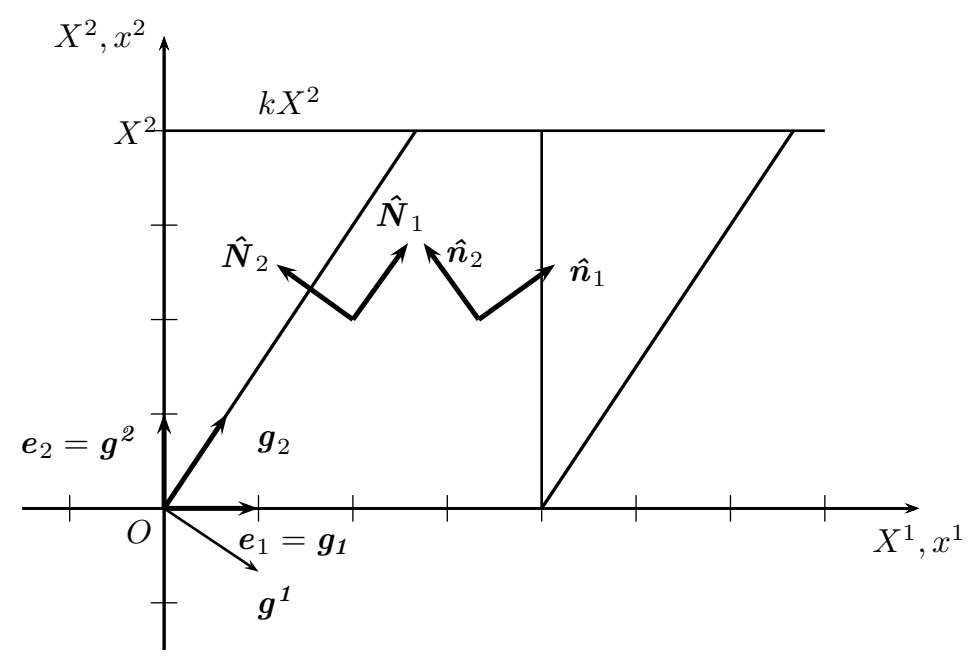

Kuva 3. Yksinkertainen leikkaus, $k=2 / 3, \theta=-18,4^{\circ}$.

Venymänopeudet ovat

$$
\begin{aligned}
\dot{\mathbf{E}}^{(2)} & =\lambda_{1} \dot{\lambda}_{1} \boldsymbol{e}_{1} \otimes \boldsymbol{e}_{1}+\lambda \dot{\lambda}\left(\boldsymbol{e}_{2} \otimes \boldsymbol{e}_{2}+\boldsymbol{e}_{3} \otimes \boldsymbol{e}_{3}\right), \\
\dot{\mathbf{e}}^{(-2)} & =\lambda_{1}^{-3} \dot{\lambda}_{1} \boldsymbol{e}_{1} \otimes \boldsymbol{e}_{1}+\lambda^{-3} \dot{\lambda}\left(\boldsymbol{e}_{2} \otimes \boldsymbol{e}_{2}+\boldsymbol{e}_{3} \otimes \boldsymbol{e}_{3}\right), \\
\dot{\mathbf{E}}^{(1)} & =\dot{\lambda}_{1} \boldsymbol{e}_{1} \otimes \boldsymbol{e}_{1}+\dot{\lambda}\left(\boldsymbol{e}_{2} \otimes \boldsymbol{e}_{2}+\boldsymbol{e}_{3} \otimes \boldsymbol{e}_{3}\right), \\
\dot{\mathbf{E}}^{(0)} & =\lambda_{1}^{-1} \dot{\lambda}_{1} \boldsymbol{e}_{1} \otimes \boldsymbol{e}_{1}+\lambda^{-1} \dot{\lambda}\left(\boldsymbol{e}_{2} \otimes \boldsymbol{e}_{2}+\boldsymbol{e}_{3} \otimes \boldsymbol{e}_{3}\right), \\
\mathbf{D} & =\frac{1}{2}\left(\mathbf{L}+\mathbf{L}^{T}\right)=\lambda_{1}^{-1} \dot{\lambda}_{1} \boldsymbol{e}_{1} \otimes \boldsymbol{e}_{1}+\lambda^{-1} \dot{\lambda}\left(\boldsymbol{e}_{2} \otimes \boldsymbol{e}_{2}+\boldsymbol{e}_{3} \otimes \boldsymbol{e}_{3}\right) .
\end{aligned}
$$

Otaksutaan, että poikittaiset jännityskomponentit $\sigma_{22}$ ja $\sigma_{33}$ ovat nollia. (Karteesisessa koordinaatistossa kovarianteilla ja kontravarianteilla komponenteilla ei ole eroa, joten kirjoitetaan jännityskomponenttien indeksit alaindekseinä). Tällöin eri venymämittoja vastaavat aksiaaliset jännitykset ovat

$$
\begin{aligned}
& \mathbf{T}^{(2)}: \dot{\mathbf{E}}^{(2)}=T_{11}^{(2)} \lambda_{1} \dot{\lambda}_{1}=\Sigma_{11} \lambda_{1}^{-1} \dot{\lambda}_{1} \quad \text { joten } \quad T_{11}^{(2)}=\Sigma_{11} / \lambda_{1}^{2}, \\
& \mathbf{T}^{(-2)}: \dot{\mathbf{E}}^{(-2)}=T_{11}^{(-2)} \lambda_{1}^{-3} \dot{\lambda}_{1}=\Sigma_{11} \lambda_{1}^{-1} \dot{\lambda}_{1} \quad \text { joten } \quad T_{11}^{(-2)}=\Sigma_{11} \lambda_{1}^{2} \text {, } \\
& \mathbf{T}^{(1)}: \dot{\mathbf{E}}^{(1)}=T_{11}^{(2)} \dot{\lambda}_{1}=\Sigma_{11} \lambda_{1}^{-1} \dot{\lambda}_{1} \quad \text { joten } \quad T_{11}^{(1)}=\Sigma_{11} / \lambda_{1}, \\
& \mathbf{T}^{(0)}: \dot{\mathbf{E}}^{(0)}=T_{11}^{(0)} \lambda_{1}^{-1} \dot{\lambda}_{1}=\Sigma_{11} \lambda_{1}^{-1} \dot{\lambda}_{1} \quad \text { joten } \quad T_{11}^{(0)}=\Sigma_{11} \text {. }
\end{aligned}
$$

Piolan-Kirchhoffin ensimmäinen jännitys saadaan vastaavasti

$$
\mathbf{P}: \dot{\mathbf{F}}=P_{11} \dot{\lambda}_{1}=\Sigma_{11} \lambda_{1}^{-1} \dot{\lambda}_{1} \quad \text { joten } P_{11}=\Sigma_{11} / \lambda_{1}=\sigma_{11} \lambda^{2}
$$

Sauvan deformoitunut poikkipinta on $A=\lambda^{2} A_{0}$, joten $P_{11}$ on nimellisjännitys ts. aksiaalinen voima jaettuna alkuperäisellä poikkipinta-alalla, kuten myös Biot'n jännitys $T_{11}^{(1)}$.

\section{Esimerkki 2. Yksinkertainen leikkaus}

Tarkastellaan muodonmuutosta, jota kuvaa yhtälö

$$
\mathbf{x}=x^{1} \mathbf{e}_{1}+x^{2} \mathbf{e}_{2}+x^{3} \mathbf{e}_{3}=\left(X^{1}+k X^{2}\right) \mathbf{e}_{1}+X^{2} \mathbf{e}_{2}+X^{3} \mathbf{e}_{3},
$$


jossa $k$ on positiivinen vakio. Kysymyksessä on $X^{1} X^{2}$-tasossa tapahtuva deformaatio, joten käsitellään sitä kaksidimensioisena. Suorakulmainen karteesinen koordinaatisto ja siihen kuuluva ortonormeerattu kanta $\left\{\mathbf{e}_{k}\right\}$ valitaan sekä alku- että lopputilan kannaksi (kuva 3). Deformaatiogradientin lauseke on

$$
\mathbf{F}=\mathbf{e}_{1} \otimes \mathbf{e}_{1}+k \mathbf{e}_{1} \otimes \mathbf{e}_{2}+\mathbf{e}_{2} \otimes \mathbf{e}_{2}
$$

Konvektiivisen koordinaatiston kovariantit kantavektorit ovat

$$
\mathbf{g}_{1}=\mathbf{e}_{1}, \quad \mathbf{g}_{2}=k \mathbf{e}_{1}+\mathbf{e}_{2}
$$

Kontravariantit vektorit ratkaistaan helposti yhtälöstä

$$
\left(\begin{array}{ll}
\mathbf{g}^{1} & \mathbf{g}^{2}
\end{array}\right)=\left(\begin{array}{ll}
\mathbf{g}_{1} & \mathbf{g}_{2}
\end{array}\right)^{-\mathrm{T}}=\left[\begin{array}{cc}
1 & 0 \\
-k & 1
\end{array}\right]
$$

josta nähdään, että ne ovat

$$
\mathbf{g}^{1}=\mathbf{e}_{1}-k \mathbf{e}_{2}, \quad \mathbf{g}^{2}=\mathbf{e}_{2}
$$

Määritetään aluksi tavanomaiset muodonmuutosmitat, jotka saadaan suoraan deformaatiogradientin (45) avulla. Käytetään tensorien asemesta 2x2-matriiseja, joiden alkiot referoidaan ortonormeeratun kannan $\left\{\mathbf{e}_{k}\right\}$ suhteen. Deformaatiogradientin matriisi, sen käänteismatriisi ja nopeusgradientin matriisi ovat

$$
\mathbf{F}=\left[\begin{array}{cc}
1 & k \\
0 & 1
\end{array}\right], \mathbf{F}^{-1}=\left[\begin{array}{cc}
1 & -k \\
0 & 1
\end{array}\right], \mathbf{L}=\left[\begin{array}{cc}
0 & \dot{k} \\
0 & 0
\end{array}\right]
$$

Cauchyn-Greenin oikeanpuoleinen muodonmuutos ja Greenin-Lagrangen venymä ovat

$$
\mathbf{C}=\mathbf{F}^{\mathrm{T}} \mathbf{F}=\left[\begin{array}{cc}
1 & k \\
k & 1+k^{2}
\end{array}\right], \mathbf{E}=\frac{1}{2}(\mathbf{C}-\mathbf{I})=\left[\begin{array}{cc}
0 & k / 2 \\
k / 2 & k^{2} / 2
\end{array}\right] .
$$

Cauchyn-Greenin vasemmanpuoleinen muodonmuutos ja Almansin-Eulerin venymä ovat

$$
\mathbf{b}=\mathbf{F F}^{\mathrm{T}}=\left[\begin{array}{cc}
1+k^{2} & k \\
k & 1
\end{array}\right], \mathbf{e}=\frac{1}{2}\left(\mathbf{I}-\mathbf{b}^{-1}\right)=\left[\begin{array}{cc}
0 & k / 2 \\
k / 2 & -k^{2} / 2
\end{array}\right] .
$$

Tasotapauksessa rotaatiotensorin matriisi on

$$
\mathbf{R}=\left[\begin{array}{cc}
\cos \theta & -\sin \theta \\
\sin \theta & \cos \theta
\end{array}\right]
$$

jolloin venytystensori $\mathbf{U}$ on

$$
\mathbf{U}=\mathbf{R}^{\mathrm{T}} \mathbf{F}=\left[\begin{array}{cc}
\cos \theta & k \cos \theta+\sin \theta \\
-\sin \theta & \cos \theta-k \sin \theta
\end{array}\right]
$$

U:n tulee olla symmetrinen, joten $k \cos \theta+\sin \theta=-\sin \theta$. Siitä seuraa $\tan \theta=-k / 2$. U:n lauseke saa silloin muodon

$$
\mathbf{U}=\left[\begin{array}{cc}
\cos \theta & -\sin \theta \\
-\sin \theta & \left(1+\sin ^{2} \theta\right) / \cos \theta
\end{array}\right]
$$


Trigonometristen suureiden paikalle voidaan sijoittaa niiden lausekkeet $k: n$ avulla lausuttuna

$$
\sin \theta=-(k / 2) / \sqrt{1+(k / 2)^{2}}, \quad \cos \theta=1 / \sqrt{1+(k / 2)^{2}} .
$$

Vastaavalla tavalla saadaan venytystensori $\mathbf{v}$

$$
\mathbf{v}=\mathbf{F R}^{\mathrm{T}}=\left[\begin{array}{cc}
\left(1+\sin ^{2} \theta\right) / \cos \theta & -\sin \theta \\
-\sin \theta & \cos \theta
\end{array}\right]
$$

Tarkastellaan sitten venymämittoja määrittelyjen (17) ja (18) mukaan. Aluksi on laskettava muodonmuutostensorin $\mathbf{C}$ ominaisarvot ja ominaisvektorit. Lausekkeen (47) avulla saadaan yhtälö

$$
\left[\begin{array}{cc}
1-\lambda^{2} & k \\
k & 1+k^{2}-\lambda^{2}
\end{array}\right]\left[\begin{array}{c}
\hat{N}_{1} \\
\hat{N}_{2}
\end{array}\right]=\left[\begin{array}{l}
0 \\
0
\end{array}\right]
$$

josta seuraa

$$
\lambda^{4}-\left(2+\lambda^{2}\right)+1=0, \quad \text { josta } \lambda_{1,2}^{2}=1+\frac{k^{2}}{2} \pm k \sqrt{1+(k / 2)^{2}}
$$

ja edelleen

$$
\lambda_{1}=\sqrt{1+(k / 2)^{2}}+k / 2=\lambda, \quad \lambda_{2}=\sqrt{1+(k / 2)^{2}}-k / 2=\lambda^{-1} .
$$

Ominaisvektoreiksi saadaan yhtälöstä (53)

$$
\hat{\boldsymbol{N}}_{1}=a \boldsymbol{e}_{1}+b \boldsymbol{e}_{2}, \quad \hat{\boldsymbol{N}}_{2}=-b \boldsymbol{e}_{1}+a \boldsymbol{e}_{2}
$$

jossa lyhenteet $a$ ja $b$ ovat

$$
a=\frac{1}{\sqrt{2}} \sqrt{1-(k / 2) / \sqrt{1+(k / 2)^{2}}}, \quad b=\frac{1}{\sqrt{2}} \sqrt{1+(k / 2) / \sqrt{1+(k / 2)^{2}}} .
$$

Vastaavasti Eulerin mukaiset (kiertyneet) ominaisvektorit ovat

$$
\hat{\boldsymbol{n}}_{1}=\mathbf{R} \hat{\boldsymbol{N}}_{1}=b \boldsymbol{e}_{1}+a \boldsymbol{e}_{2}, \quad \hat{\boldsymbol{n}}_{2}=\mathbf{R} \hat{\boldsymbol{N}}_{2}=-a \boldsymbol{e}_{1}+b \boldsymbol{e}_{2}
$$

Jos ominaisarvot ja ominaisvektorit lausutaan kulman $\theta$ funktioina, saadaan

$$
\begin{gathered}
\lambda_{1}=\sqrt{\frac{1-\sin \theta}{1+\sin \theta}}, \quad \lambda_{2}=\sqrt{\frac{1+\sin \theta}{1-\sin \theta}}=\lambda_{1}^{-1} \\
a=\frac{1}{\sqrt{2}} \sqrt{1+\sin \theta}, \quad b=\frac{1}{\sqrt{2}} \sqrt{1-\sin \theta} .
\end{gathered}
$$

Kirjoitetaan sitten muodonmuutosmittojen lausekkeet, joissa matriisien alkiot ovat kannan $\left\{\mathbf{e}_{k}\right\}$ suhteen,

$$
\begin{aligned}
\mathbf{E}^{(2)} & =\frac{1}{2}\left[\left(\lambda^{2}-1\right) \hat{\boldsymbol{N}}_{1} \otimes \hat{\boldsymbol{N}}_{1}+\left(\lambda^{-2}-1\right) \hat{\mathbf{N}}_{2} \otimes \hat{\mathbf{N}}_{2}\right] \\
{\left[\mathbf{E}^{(2)}\right] } & =\frac{b^{2}-a^{2}}{2 a b}\left[\begin{array}{cc}
0 & 1 \\
1 & \left(b^{2}-a^{2}\right) / a b
\end{array}\right]
\end{aligned}
$$




$$
\begin{aligned}
& \mathbf{E}^{(-2)}=\frac{1}{2}\left[\left(1-\lambda^{-2}\right) \hat{\boldsymbol{N}}_{1} \otimes \hat{\boldsymbol{N}}_{1}+\left(1-\lambda^{2}\right) \hat{\mathbf{N}}_{2} \otimes \hat{\boldsymbol{N}}_{2}\right] \\
& {\left[\mathbf{E}^{(-2)}\right]=\frac{b^{2}-a^{2}}{2 a b}\left[\begin{array}{cc}
\left(a^{2}-b^{2}\right) / a b & 1 \\
1 & 0
\end{array}\right] \text {, }} \\
& \mathbf{e}^{(-2)}=\mathbf{R E}^{(-2)} \mathbf{R}^{\mathrm{T}}, \quad\left[\mathbf{e}^{(-2)}\right]=\frac{b^{2}-a^{2}}{2 a b}\left[\begin{array}{cc}
0 & 1 \\
1 & \left(a^{2}-b^{2}\right) / a b
\end{array}\right], \\
& \mathbf{E}^{(1)}=\left[(\lambda-1) \hat{\boldsymbol{N}}_{1} \otimes \hat{\boldsymbol{N}}_{1}+\left(\lambda^{-1}-1\right) \hat{\boldsymbol{N}}_{2} \otimes \hat{\boldsymbol{N}}_{2}\right] \\
& {\left[\mathbf{E}^{(1)}\right]=(b-a)\left[\begin{array}{cc}
a-b & a+b \\
a+b & (b-a)(1+a b) / a b
\end{array}\right],} \\
& \mathbf{e}^{(1)}=\left[(\lambda-1) \hat{\boldsymbol{n}}_{1} \otimes \hat{\boldsymbol{n}}_{1}+\left(\lambda^{-1}-1\right) \hat{\boldsymbol{n}}_{2} \otimes \hat{\boldsymbol{n}}_{2}\right], \\
& {\left[\mathbf{e}^{(1)}\right]=(b-a)\left[\begin{array}{cc}
(b-a)(1+a b) / a b & a+b \\
a+b & a-b
\end{array}\right],} \\
& \ln \mathbf{U}=\ln \lambda \hat{\boldsymbol{N}}_{1} \otimes \hat{\boldsymbol{N}}_{1}-\ln \lambda \hat{\boldsymbol{N}}_{2} \otimes \hat{\boldsymbol{N}}_{2}, \quad[\ln \mathbf{U}]=\ln \lambda\left[\begin{array}{cc}
a^{2}-b^{2} & 2 a b \\
2 a b & b^{2}-a^{2}
\end{array}\right] \\
& \ln \mathbf{v}=\ln \lambda \hat{\boldsymbol{n}}_{1} \otimes \hat{\boldsymbol{n}}_{1}-\ln \lambda \hat{\boldsymbol{n}}_{2} \otimes \hat{\boldsymbol{n}}_{2}, \quad[\ln \mathbf{v}]=\ln \lambda\left[\begin{array}{cc}
b^{2}-a^{2} & 2 a b \\
2 a b & a^{2}-b^{2}
\end{array}\right] .
\end{aligned}
$$

Ominaisvektorit antavat päävenymien suunnat. Lagrangen mukaiset suunnat $X^{1}$-akselin suhteen ovat

$$
\cos \psi_{1}^{L}=\hat{\boldsymbol{N}}_{1} \cdot \boldsymbol{e}_{1}=\frac{1}{\sqrt{2}} \sqrt{1+\sin \theta}, \quad \cos \psi_{2}^{L}=\hat{\boldsymbol{N}}_{2} \cdot \boldsymbol{e}_{1}=-\frac{1}{\sqrt{2}} \sqrt{1-\sin \theta}
$$

Vastaavasti Eulerin mukaiset päävenymäsuunnat ovat

$$
\cos \psi_{1}^{E}=\hat{\boldsymbol{n}}_{1} \cdot \boldsymbol{e}_{1}=\frac{1}{\sqrt{2}} \sqrt{1-\sin \theta}, \quad \cos \psi_{2}^{E}=\hat{\boldsymbol{n}}_{2} \cdot \boldsymbol{e}_{1}=-\frac{1}{\sqrt{2}} \sqrt{1+\sin \theta} .
$$

Venymänopeudet saadaan kaavoista (47), (48), (46), (50) ja (52) derivoimalla ajan suhteen

$$
\begin{gathered}
\dot{\mathbf{E}}=\left[\begin{array}{cc}
0 & \dot{k} / 2 \\
\dot{k} / 2 & k \dot{k}
\end{array}\right], \quad \dot{\mathbf{e}}=\left[\begin{array}{cc}
0 & \dot{k} / 2 \\
\dot{k} / 2 & -k \dot{k}
\end{array}\right], \quad \mathbf{D}=\left[\begin{array}{cc}
0 & \dot{k} / 2 \\
\dot{k} / 2 & 0
\end{array}\right] \\
\dot{\mathbf{U}}=\dot{\theta}\left[\begin{array}{cc}
-\sin \theta & -\cos \theta \\
-\cos \theta & \sin \theta\left(1+2 / \cos ^{2} \theta\right)
\end{array}\right], \quad \dot{\mathbf{v}}=\dot{\theta}\left[\begin{array}{cc}
\sin \theta\left(1+2 / \cos ^{2} \theta\right) & -\cos \theta \\
-\cos \theta & -\sin \theta
\end{array}\right] \\
(\ln \mathbf{U})^{\bullet}=\frac{\dot{\lambda}_{1}}{\lambda_{1}}\left[\begin{array}{cc}
\sin \theta & \cos \theta \\
\cos \theta & -\sin \theta
\end{array}\right]+\dot{\theta} \ln \lambda_{1}\left[\begin{array}{cc}
\cos \theta & -\sin \theta \\
-\sin \theta & -\cos \theta
\end{array}\right] \\
(\ln \mathbf{v})^{\bullet}=\frac{\dot{\lambda}_{1}}{\lambda_{1}}\left[\begin{array}{cc}
-\sin \theta & \cos \theta \\
\cos \theta & \sin \theta
\end{array}\right]+\dot{\theta} \ln \lambda_{1}\left[\begin{array}{cc}
-\cos \theta & -\sin \theta \\
-\sin \theta & \cos \theta
\end{array}\right]
\end{gathered}
$$

Kiertymänopeudet kaavojen (22), (23) ja (25) mukaan ovat

$$
\begin{aligned}
& {\left[\boldsymbol{\Omega}^{(\mathrm{R})}\right]=[\dot{\mathbf{R}}][\mathbf{R}]^{\mathrm{T}}=\dot{\theta}\left[\begin{array}{cc}
-\sin \theta & -\cos \theta \\
\cos \theta & -\sin \theta
\end{array}\right]\left[\begin{array}{cc}
\cos \theta & \sin \theta \\
-\sin \theta & \cos \theta
\end{array}\right]=\dot{\theta}\left[\begin{array}{cc}
0 & -1 \\
1 & 0
\end{array}\right],} \\
& {\left[\boldsymbol{\Omega}^{(\mathrm{L})}\right]=\left[\dot{\mathbf{R}}^{(\mathrm{L})}\right]\left[\mathbf{R}^{(\mathrm{L})}\right]^{\mathrm{T}}=\left[\begin{array}{cc}
\dot{a} & -\dot{b} \\
\dot{b} & \dot{a}
\end{array}\right]\left[\begin{array}{cc}
a & b \\
-b & a
\end{array}\right]=\frac{\dot{\theta}}{2}\left[\begin{array}{cc}
0 & 1 \\
-1 & 0
\end{array}\right],} \\
& {\left[\boldsymbol{\Omega}^{(\mathrm{E})}\right]=\left[\boldsymbol{\Omega}^{(\mathrm{R})}\right]+[\mathbf{R}]\left[\boldsymbol{\Omega}^{(\mathrm{L})}\right][\mathbf{R}]^{\mathrm{T}}=\frac{\dot{\theta}}{2}\left[\begin{array}{cc}
0 & -1 \\
1 & 0
\end{array}\right] .}
\end{aligned}
$$


Muodostetaan sitten venymänopeuksien lausekkeet käyttäen esitystapaa (17) ja (18). Yhtälöistä (59) seuraa

$$
\begin{aligned}
\dot{\mathbf{E}}^{(2)} & =\frac{\dot{\theta}}{4 a^{3} b^{3}}\left[-2 b^{4} \hat{\boldsymbol{N}}_{1} \otimes \hat{\boldsymbol{N}}_{1}+2 a^{4} \hat{\boldsymbol{N}}_{2} \otimes \hat{\boldsymbol{N}}_{2}+a b\left(a^{2}-b^{2}\right)\left(\hat{\boldsymbol{N}}_{1} \otimes \hat{\boldsymbol{N}}_{2}+\hat{\boldsymbol{N}}_{2} \otimes \hat{\boldsymbol{N}}_{1}\right)\right], \\
\dot{\mathbf{E}}^{(-2)} & =\frac{\dot{\theta}}{4 a^{3} b^{3}}\left[-2 a^{4} \hat{\boldsymbol{N}}_{1} \otimes \hat{\boldsymbol{N}}_{1}+2 b^{4} \hat{\boldsymbol{N}}_{2} \otimes \hat{\boldsymbol{N}}_{2}+a b\left(a^{2}-b^{2}\right)\left(\hat{\boldsymbol{N}}_{1} \otimes \hat{\boldsymbol{N}}_{2}+\hat{\boldsymbol{N}}_{2} \otimes \hat{\boldsymbol{N}}_{1}\right)\right] \\
\dot{\mathbf{e}}^{(-2)} & =\frac{\dot{\theta}}{4 a^{3} b^{3}}\left[-2 a^{4} \hat{\boldsymbol{n}}_{1} \otimes \hat{\boldsymbol{n}}_{1}+2 b^{4} \hat{\boldsymbol{n}}_{2} \otimes \hat{\boldsymbol{n}}_{2}-a b\left(a^{2}-b^{2}\right)\left(\hat{\boldsymbol{n}}_{1} \otimes \hat{\boldsymbol{n}}_{2}+\hat{\boldsymbol{n}}_{2} \otimes \hat{\boldsymbol{n}}_{1}\right)\right], \\
\dot{\mathbf{U}} & =\dot{\mathbf{E}}^{(1)}=\frac{\dot{\theta}}{2 a^{2} b^{2}}\left[-b^{2} \hat{\boldsymbol{N}}_{1} \otimes \hat{\boldsymbol{N}}_{1}+a^{2} \hat{\boldsymbol{N}}_{2} \otimes \hat{\boldsymbol{N}}_{2}+a b\left(a^{2}-b^{2}\right)\left(\hat{\boldsymbol{N}}_{1} \otimes \hat{\boldsymbol{N}}_{2}+\hat{\boldsymbol{N}}_{2} \otimes \hat{\boldsymbol{N}}_{1}\right)\right], \\
\dot{\mathbf{v}} & =\dot{\mathbf{e}}^{(1)}=\frac{\dot{\theta}}{2 a^{2} b^{2}}\left[-b^{2} \hat{\boldsymbol{n}}_{1} \otimes \hat{\boldsymbol{n}}_{1}+a^{2} \hat{\boldsymbol{n}}_{2} \otimes \hat{\boldsymbol{n}}_{2}-a b\left(a^{2}-b^{2}\right)\left(\hat{\boldsymbol{n}}_{1} \otimes \hat{\boldsymbol{n}}_{2}+\hat{\boldsymbol{n}}_{2} \otimes \hat{\boldsymbol{n}}_{1}\right)\right] .
\end{aligned}
$$

Kun näihin sijoitetaan ominaisvektorien lausekkeet (56) ja (58), saadaan venymänopeudet (62) ja (63). Venymänopeus D on kaavan (27) mukaan

$$
\mathbf{D}=\frac{\dot{\theta}}{4 a^{2} b^{2}}\left[2 a b\left(-\hat{\boldsymbol{n}}_{1} \otimes \hat{\boldsymbol{n}}_{1}+\hat{\boldsymbol{n}}_{2} \otimes \hat{\boldsymbol{n}}_{2}\right)+\left(a^{2}-b^{2}\right)\left(\hat{\boldsymbol{n}}_{1} \otimes \hat{\boldsymbol{n}}_{2}+\hat{\boldsymbol{n}}_{2} \otimes \hat{\boldsymbol{n}}_{1}\right)\right]
$$

mikä taas sijoittamalla ominaisvektorit $\hat{\boldsymbol{n}}_{k}$ saa muodon $(62)_{3}$. Logaritmiset venymänopeudet saadaan kaavoista $(59)_{6}$ ja $(59)_{7}$

$$
\begin{aligned}
(\ln \mathbf{U})^{\bullet} & =\dot{\theta}\left[\frac{1}{2 a b}\left(-\hat{\boldsymbol{N}}_{1} \otimes \hat{\boldsymbol{N}}_{1}+\hat{\boldsymbol{N}}_{2} \otimes \hat{\boldsymbol{N}}_{2}\right)-\ln \frac{b}{a}\left(\hat{\boldsymbol{N}}_{1} \otimes \hat{\boldsymbol{N}}_{2}+\hat{\boldsymbol{N}}_{2} \otimes \hat{\boldsymbol{N}}_{1}\right)\right] \\
(\ln \mathbf{v})^{\bullet} & =\dot{\theta}\left[\frac{1}{2 a b}\left(-\hat{\boldsymbol{n}}_{1} \otimes \hat{\boldsymbol{n}}_{1}+\hat{\boldsymbol{n}}_{2} \otimes \hat{\boldsymbol{n}}_{2}\right)+\ln \frac{b}{a}\left(\hat{\boldsymbol{n}}_{1} \otimes \hat{\boldsymbol{n}}_{2}+\hat{\boldsymbol{n}}_{2} \otimes \hat{\boldsymbol{n}}_{1}\right)\right] .
\end{aligned}
$$

Sijoittamalla näihin ominaisvektorien lausekkeet saadaan taas venymänopeuksien kaavat (64) ja (65). Matriisimuodossa edellä olevat lausekkeet ovat

$$
\begin{aligned}
& {\left[\dot{\mathbf{E}}^{(2)}\right]=\frac{\dot{\theta}}{4 a^{3} b^{3}}\left[\begin{array}{cc}
0 & -a b \\
-a b & 2\left(a^{2}-b^{2}\right)
\end{array}\right]} \\
& {\left[\dot{\mathbf{E}}^{(-2)}\right]=\frac{\dot{\theta}}{4 a^{3} b^{3}}\left[\begin{array}{cc}
2\left(b^{2}-a^{2}\right) & -a b \\
-a b & 0
\end{array}\right]} \\
& {\left[\dot{\mathbf{e}}^{(-2)}\right]=\frac{\dot{\theta}}{4 a^{3} b^{3}}\left[\begin{array}{cc}
0 & -a b \\
-a b & 2\left(b^{2}-a^{2}\right)
\end{array}\right],} \\
& {[\dot{\mathbf{U}}]=\left[\dot{\mathbf{E}}^{(1)}\right]=\dot{\theta}\left[\begin{array}{cc}
\left(b^{2}-a^{2}\right) & -2 a b \\
-2 a b & \left(a^{2}-b^{2}\right)\left(1+2 a^{2} b^{2}\right) / 2 a^{2} b^{2}
\end{array}\right],} \\
& {[\dot{\mathbf{v}}]=\left[\dot{\mathbf{e}}^{(1)}\right]=\dot{\theta}\left[\begin{array}{cc}
\left(a^{2}-b^{2}\right)\left(1+2 a^{2} b^{2}\right) / 2 a^{2} b^{2} & -2 a b \\
-2 a b & \left(a^{2}-b^{2}\right)
\end{array}\right],} \\
& {[\mathbf{D}]=\frac{\dot{\theta}}{4 a^{2} b^{2}}\left[\begin{array}{cc}
0 & -1 \\
-1 & 0
\end{array}\right],} \\
& {\left[(\ln \mathbf{U})^{\bullet}\right]=\dot{\theta}\left\{\frac{1}{2 a b}\left[\begin{array}{cc}
b^{2}-a^{2} & -2 a b \\
-2 a b & a^{2}-b^{2}
\end{array}\right]+\ln \frac{b}{a}\left[\begin{array}{cc}
2 a b & b^{2}-a^{2} \\
b^{2}-a^{2} & -2 a b
\end{array}\right]\right\},}
\end{aligned}
$$




$$
\left[(\ln \mathbf{v})^{\bullet}\right]=\dot{\theta}\left\{\frac{1}{2 a b}\left[\begin{array}{cc}
a^{2}-b^{2} & -2 a b \\
-2 a b & b^{2}-a^{2}
\end{array}\right]+\ln \frac{b}{a}\left[\begin{array}{cc}
-2 a b & b^{2}-a^{2} \\
b^{2}-a^{2} & 2 a b
\end{array}\right]\right\} .
$$

Muodostetaan vielä alkutilassa lausuttujen jännitysten lausekkeet Kirchhoffin (tai Cauchyn) jännitysten funktioina. Kirjoitetaan jännitysten indeksit alaindekseinä, koska ne referoidaan suorakulmaiseen karteesiseen kantajärjestelmään. Käytetään hyväksi kaavoja (37),(39),(41),(44) ja (42). Piolan-Kirchhoffin toiselle jännitykselle saadaan lausekkeet

$$
T_{11}^{(2)}=\frac{a^{2}}{b^{2}} \Sigma_{11}, \quad T_{22}^{(2)}=\frac{b^{2}}{a^{2}} \Sigma_{22}, \quad T_{12}^{(2)}=\Sigma_{12}=T_{21}^{(2)}=\Sigma_{21} .
$$

Määritetään Piolan-Kirchhoffin jännityskomponentit kiinteän kannan $\left\{\mathbf{e}_{k}\right\}$ suhteen. Jännitystensori on

$$
T_{k l}^{(2)} \hat{\boldsymbol{N}}_{k} \otimes \hat{\boldsymbol{N}}_{l}=S_{r s}^{(2)} \boldsymbol{e}_{r} \otimes \boldsymbol{e}_{s}
$$

Komponenttien välinen muunnos seuraa ominaisvektorien kaavoista (56)

$$
\left[\begin{array}{ll}
S_{11}^{(2)} & S_{12}^{(2)} \\
S_{21}^{(2)} & S_{22}^{(2)}
\end{array}\right]=\left[\begin{array}{cc}
a & -b \\
b & a
\end{array}\right]\left[\begin{array}{cc}
T_{11}^{(2)} & T_{12}^{(2)} \\
T_{21}^{(2)} & T_{22}^{(2)}
\end{array}\right]\left[\begin{array}{cc}
a & -b \\
b & a
\end{array}\right]^{\mathrm{T}}
$$

Samantapainen muunnos tehdään Kirchhoffin jännitykselle

$$
\Sigma_{k l} \hat{\boldsymbol{n}}_{k} \otimes \hat{\boldsymbol{n}}_{l}=J \sigma_{r s} \boldsymbol{e}_{r} \otimes \boldsymbol{e}_{s}
$$

Ominaisvektorien (58) avulla saadaan komponenttien muunnoskaava

$$
\left[\begin{array}{cc}
\Sigma_{11} & \Sigma_{12} \\
\Sigma_{21} & \Sigma_{22}
\end{array}\right]=J\left[\begin{array}{cc}
b & -a \\
a & b
\end{array}\right]^{\mathrm{T}}\left[\begin{array}{ll}
\sigma_{11} & \sigma_{12} \\
\sigma_{21} & \sigma_{22}
\end{array}\right]\left[\begin{array}{cc}
b & -a \\
a & b
\end{array}\right]
$$

Muunnoksien jälkeen saadaan, ottaen huomioon, että tässä tapauksessa $J=1$,

$$
\begin{gathered}
S_{11}^{(2)}=\sigma_{11}+\frac{a^{2}-b^{2}}{a b}\left(\sigma_{12}+\sigma_{21}\right)+\frac{\left(a^{2}-b^{2}\right)^{2}}{a^{2} b^{2}} \sigma_{22}, \quad S_{12}^{(2)}=\sigma_{12}+\frac{a^{2}-b^{2}}{a b} \sigma_{22} \\
S_{21}^{(2)}=\sigma_{21}+\frac{a^{2}-b^{2}}{a b} \sigma_{22}, \quad S_{22}^{(2)}=\sigma_{22} .
\end{gathered}
$$

Vastaavasti menettelemällä saadaan Almansin jännitykselle aluksi

$$
T_{11}^{(-2)}=\frac{b^{2}}{a^{2}} \Sigma_{11}, \quad T_{22}^{(-2)}=\frac{a^{2}}{b^{2}} \Sigma_{22}, \quad T_{12}^{(-2)}=\Sigma_{12}=T_{21}^{(-2)}=\Sigma_{21},
$$

ja sen jälkeen

$$
\begin{gathered}
S_{11}^{(-2)}=\sigma_{11}, \quad S_{12}^{(-2)}=\sigma_{12}+\frac{b^{2}-a^{2}}{a b} \sigma_{11} \\
S_{21}^{(-2)}=\sigma_{21}+\frac{b^{2}-a^{2}}{a b} \sigma_{11}, \quad S_{22}^{(-2)}=\sigma_{22}+\frac{b^{2}-a^{2}}{a b}\left(\sigma_{12}+\sigma_{21}\right)+\frac{\left(a^{2}-b^{2}\right)^{2}}{a^{2} b^{2}} \sigma_{11} .
\end{gathered}
$$

Biot'n jännityksen symmetriselle osalle saadaan vastaavasti menettelemällä

$$
T_{11}^{(1)}=\frac{a}{b} \Sigma_{11}, \quad T_{22}^{(1)}=\frac{b}{a} \Sigma_{22}, \quad T_{12}^{(1)}=\frac{1}{2 a b} \Sigma_{12}=T_{21}^{(1)}=\frac{1}{2 a b} \Sigma_{21},
$$




$$
\begin{aligned}
& S_{11}^{(1)}=2 a b \sigma_{11}+\frac{3\left(a^{2}-b^{2}\right)}{2}\left(\sigma_{12}+\sigma_{21}\right)+\frac{\left(a^{2}-b^{2}\right)^{2}}{a b} \sigma_{22}, \\
& S_{12}^{(1)}=\frac{b^{2}-a^{2}}{2} \sigma_{11}+2 a b \sigma_{12}-\frac{\left(a^{2}-b^{2}\right)^{2}}{2 a b} \sigma_{21}+\frac{3\left(a^{2}-b^{2}\right)}{2} \sigma_{22}, \\
& S_{21}^{(1)}=\frac{b^{2}-a^{2}}{2} \sigma_{11}-\frac{\left(a^{2}-b^{2}\right)^{2}}{2 a b} \sigma_{12}+2 a b \sigma_{21}+\frac{3\left(a^{2}-b^{2}\right)}{2} \sigma_{22}, \\
& S_{22}^{(1)}=2 a b \sigma_{22}+\frac{b^{2}-a^{2}}{2}\left(\sigma_{12}+\sigma_{21}\right) .
\end{aligned}
$$

Lopuksi Henckyn jännitys saa melkoisen komplisoidut lausekkeet

$$
\begin{aligned}
& T_{11}^{(0)}=\Sigma_{11}, \quad T_{22}^{(0)}=\Sigma_{22}, \quad T_{12}^{(0)}=\frac{a^{2}-b^{2}}{4 a^{2} b^{2} \ln (a / b)} \Sigma_{12}=T_{21}^{(0)}=\frac{a^{2}-b^{2}}{4 a^{2} b^{2} \ln (a / b)} \Sigma_{21}, \\
& S_{11}^{(0)}=\left[1+\frac{a^{2}-b^{2}}{4 a^{2} b^{2} \ln (a / b)}\right]\left[2 a^{2} b^{2}\left(\sigma_{11}-\sigma_{22}\right)+a b\left(a^{2}-b^{2}\right)\left(\sigma_{12}+\sigma_{21}\right)\right]+\sigma_{22}, \\
& S_{12}^{(0)}=\left[1+\frac{a^{2}-b^{2}}{4 a^{2} b^{2} \ln (a / b)}\right]\left[a b\left(a^{2}-b^{2}\right)\left(\sigma_{22}-\sigma_{11}\right)+2 a^{2} b^{2}\left(\sigma_{12}+\sigma_{21}\right)-\sigma_{21}\right]+\sigma_{21}, \\
& S_{21}^{(0)}=\left[1+\frac{a^{2}-b^{2}}{4 a^{2} b^{2} \ln (a / b)}\right]\left[a b\left(a^{2}-b^{2}\right)\left(\sigma_{22}-\sigma_{11}\right)+2 a^{2} b^{2}\left(\sigma_{12}+\sigma_{21}\right)-\sigma_{12}\right]+\sigma_{12}, \\
& S_{22}^{(0)}=\left[1+\frac{a^{2}-b^{2}}{4 a^{2} b^{2} \ln (a / b)}\right]\left[2 a^{2} b^{2}\left(\sigma_{22}-\sigma_{11}\right)-a b\left(a^{2}-b^{2}\right)\left(\sigma_{12}+\sigma_{21}\right)\right]+\sigma_{11} .
\end{aligned}
$$

Kirjoitetaan vielä Piolan-Kirchhoffin ensimmäisen jännityksen vastaavat lausekkeet

$$
\begin{aligned}
& \mathbf{P}^{(1)}=\sum_{k, l=1}^{2} P_{k l}^{(1)} \hat{\boldsymbol{n}}_{k} \otimes \hat{\boldsymbol{N}}_{l}, \quad\left[\begin{array}{cc}
P_{11}^{(1)} & P_{12}^{(1)} \\
P_{21}^{(1)} & P_{22}^{(1)}
\end{array}\right]= \\
& =\frac{1}{a b}\left[\begin{array}{cc}
a^{2} b^{2} \sigma_{11}+a^{3} b\left(\sigma_{12}+\sigma_{21}\right)+a^{4} \sigma_{22} & -a b^{3}\left(\sigma_{11}-\sigma_{22}\right)+b^{4} \sigma_{12}-a^{2} b^{2} \sigma_{21} \\
-a^{3} b\left(\sigma_{11}-\sigma_{22}\right)-a^{4} \sigma_{12}+a^{2} b^{2} \sigma_{21} & a^{2} b^{2} \sigma_{11}-a b^{3}\left(\sigma_{12}+\sigma_{21}\right)+b^{4} \sigma_{22}
\end{array}\right]
\end{aligned}
$$

Kun matriisin alkiot ovat kannan $\left\{\mathbf{e}_{k}\right\}$ suhteen, edellä olevasta saadaan

$$
\left[\begin{array}{ll}
P_{11} & P_{12} \\
P_{21} & P_{22}
\end{array}\right]=\frac{1}{a b}\left[\begin{array}{cc}
a b \sigma_{11}+\left(a^{2}-b^{2}\right) \sigma_{12} & \sigma_{12} \\
\left(a^{2}-b^{2}\right) \sigma_{22}+a b \sigma_{21} & a b \sigma_{22}
\end{array}\right], \quad \text { ja } \quad[\dot{\mathbf{F}}]=\frac{\dot{\theta}}{2 a^{2} b^{2}}\left[\begin{array}{cc}
0 & -1 \\
0 & 0
\end{array}\right] .
$$

Edellä esitetyistä jännitysten ja venymänopeuksien lausekkeista lasketut teholausekkeet ovat tietenkin yhtäsuuret ts. niillä on arvo $\dot{W}=\sigma_{k l} d_{k l}=\sigma_{12} \dot{k}$, joka oli niiden määritysehto.

\section{Yhteenveto}

Artikkelissa on tarkasteltu kontinuumimekaniikan tavallisimpia venymämittoja R.Hillin ja B.R.Sethin esittämän yleisen kaavan perusteella mukaan lukien H.Henckyn logaritminen venymä. On johdettu venymänopeuksien kaavat ja määritetty venymämittoja vastaavat jännitysmitat. Esimerkkeinä on käsitelty vedettyä sauvaa ja yksinkertaista leikkausta. Eri jännitysmittojen lausekkeet Cauchyn jännityksen funktioina on johdettu. 


\section{Viitteet}

[1] M.E. Gurtin and K. Spear. On the relationship between the logarithmic strain rate and the stretching tensor. International Journal of Solids and Structures, 19:437-444, 1983. doi:10.1016/0020-7683(83)90054-9.

[2] H. Hencky. Über die Form des Elastizitätsgesetzes bei ideal elastischen Stoffen. Zeitschrift für Technische Physik, 9:215-220, 457, 1928.

[3] R. Hill. Aspects of invariance in solid mechanics. Advances in Applied Mechanics, Vol. 18:1-75, 1978. doi:10.1016/S0065-2156(08)70264-3.

[4] A. Hoger. The material time derivative of logarithmic strain. International Journal of Solids and Structures, 22(9):1019-1032, 1986. doi:10.1016/0020-7683(86)90034-X.

[5] A. Hoger. The stress conjugate to logarithmic strain. International Journal of Solids and Structures, 23(12):1645-1656, 1987. doi:10.1016/0020-7683(87)90115-6.

[6] G.A. Holzapfel. Nonlinear Solid Mechanics - A Continuum Approach for Engineering. John Wiley \& Sons, 2000.

[7] D.B. Macvean. Elementararbeit in einem Kontinuum und die Zuordnung von Spannungs- und Verzerrungstensoren. Zeitschrift für Angewandte Mathematik und Physik, 19:157-185, 1968. doi:10.1007/BF01601465.

[8] B.R. Seth. Generalized strain measure with applications to physical problems. In M. Reiner and D. Abir, editors, Second-Order Effects in Elasticity, Plasticity and Fluid Dynamics,IUTAM Symposium, Haifa, Israel, 23-27.4.1963, 1964.

Martti Mikkola

Aalto-yliopisto

Rakennustekniikan laitos

PL 12100, 00076 Aalto

martti.mikkola@aalto.fi 